\title{
ÜBER REZENTE ERDRUTSCHE UND FELSSTÜRZE IN DER SCHWEIZ
}

\author{
Fritz Nussbaum
}

Bei ihren Untersuchungen über die geologische Beschaffenheit unseres Landes stellten viele Forscher das Vorkommen von Schuttbildungen fest, die sich infolge der mechanischen Verwitterung in den Gebirgen teils als Berg- und Felsstürze, sowie als Schutthalden aufgehäuft haben oder die teils als Erdrutsche, Rüfen, Murgänge und Wildbachausbrüche zu erkennen sind.

Im besonderen haben A. Heim, A. Baltzer, E. Brückner, Oberholzer, J. Früh, H. Schardi, M. Lugeon, A. Buxtorf, V. Gilliéron, J. Cadisch und ihre Schüler die Vorgänge und Ablagerungen von Bergstürzen geschildert, die sich teils in früheren Epochen, teils in neuerer Zeit abgespielt haben. Bei verschiedenen rezenten Bergstürzen ist auch der Einfluß von starken Regenfällen auf die zerklüfteten, durchlässigen Felsschichten betont worden, so von A. HEIM, J. FRÜH u. a.

Solche Ereignisse wurden in den letzten Jahren auch von RaOul und Fred. MoNTandon in den "Matériaux pour l'étude des Calamités» besprochen (Lit. 12, 13, 14).

Schließlich sei darauf hingewiesen, daß die Tagespresse häufig teils ausführliche, teils kurz gehaltene Mitteilungen über verschiedene Arten von Erdbewegungen gebracht hat. Auf Grund der in den vergangenen drei Jahrzehnten erfolgten Berichte hat der Verfasser eine Zusammenstellung der in dieser Zeit in der Schweiz vorgekommenen Bodenbewegungen, wie Erdrutsche, Fels- und Bergstürze, gemacht (deren Zahl über 540 beträgt) und berichtet hierüber in aller Kürze.

Zweifellos umfaßt diese Zahl nicht die sämtlichen während 30 Jahren erfolgten Erdbewegungen unseres Landes; denn wie A. HeIM und J. FRÜH ausgeführt haben, können je nach den Untergrundund Niederschlagsverhältnissen am gleichen Ort gleichzeitig mehrere Rutschungen erfolgen. In unseren Zahlen sind eigentlich die Orte verstanden, an denen gleichen Tages eine oder mehrere Rutschungen erfolgt sind.

JURA

- Im Gebiet des Schweizer Juras ereigneten sich in den letzten 30 Jahren 44 Fälle von Erdbewegungen, von denen sich die meisten an 38 Regentagen abspielten. Es lassen sich hierbei 4 Felsstürze und 40 Rutsche, Schlipfe und Murgänge unterscheiden. Solche kamen hauptsächlich in den weit verbreiteten tonigen Oxford- und Effingerschichten vor. Einen großen Umfang erreichten solche Ausbrüche im März und April 1937 in der Klus von Court; sie sind von mehreren sachkundigen Autoren ausführlich beschrieben worden (Lit. 3, 18, 20). Andere bedeutende Rutsche ereigneten sich an den folgenden Orten: Im März 1931 bei Eptingen, im März 1937 bei Twann, im Juni 1938 bei Bärschwil, im April 1952 am Wartenberg bei Muttenz und mehrmals, nämlich 1944, 1946 und 1956 im Traverstal (Lit. 21, 22, 5, p, 205).

\section{MITTELLAND}

Im Mittelland fanden während der Beobachtungszeit an 99 Orten Erdbewegungen statt, die sich auf 89 Rutschungen und 10 Felsstürze verteilen. Ihr Hauptgebiet ist das dem Alpenrand benachbarte höhere und reich durchtalte Mittelland, das abwechselnd aus Sandsteinen, Mergel- und Nagelfluhbänken der Molasse besteht. Zufolge häufiger Regen kommt es in dem aufgeweichten Boden an den relativ steilen Hängen an vielen Orten zu Rutschungen, durch welche an mehreren Stellen auch Siedlungen betroffen werden und sogar Menschen den Tod gefunden haben. Die Gesamtzahl der Regentage in Mittelland betrug 81 .

In der Westschweiz, zwischen Aare und Genfersee, wurden 18 Rutsche und 3 Felsstürze festgestellt. Größere Rutschungen fanden in der Umgebung von Lausanne und bei Vevey statt, so 1936 bei Belmont, 1950 bei Chexbres und 1954 bei Pully. Bei Vevey, wo bereits 1877 ein Quaiabsturz stattgefunden hatte, wiederholte sich dieser Vorgang im Jahr 1933.

Im bernisch-luzernischen Mittelland wurden an 34 Orten Erdbewegungen beobachtet, von denen sich die meisten in den Einzugsgebieten der beiden Emmen ereigneten. 2 Felsstürzen stehen 32 Rutschungen und Wildbachausbrüche gegenüber. Von letzteren wurden namentlich das obere Emmental mit seinen vom Napfgebiet herab- 
führenden Tälern heimgesucht, so insbesondere die Umgebungen von Trub, Langnau, Signau, Wasen und weitere Gebiete. Ebenso kamen Verheerungen im Entlebuch, bei Malters und an der Schwarzenegg vor. Felsstürze ereigneten sich bei Burgdorf und bei Gümmenen.

In den Gebieten der Kantone Zürich, Thurgau, St. Gallen und Appenzell A./Rh. wurden in der angegebenen Zeit 28 Ortschaften durch Rutschungen und Felsstürze heimgesucht. An mehreren Orten fanden infolge starker Regen gleichen Tags mehrere Rutschungen statt, so bei Stettfurt, Herisau, Teufen, Lütisburg, Wattwil, bei Wald und bei Ermatingen. Größere Rutsche ereigneten sich bei Rorschach (1935), bei Untereggen (1936), und Felsstürze kamen bei Lütisburg (1935) und bei Kempelbach (1939) vor.

\section{ERD- UND FELSBEWEGUNGEN IN DEN NORDALPEN}

Bekanntlich unterliegen die Ketten und Gipfel der Hochgebirge einer starken mechanischen Verwitterung und sind daher durch ausgesprochene Häufigkeit von Fels- und Bergstürzen gekennzeichnet. Da unsere Alpen gleichzeitig häufige und reichliche Niederschläge erhalten, kommen an den steilen Berghängen zahlreiche Rutschungen, Murgänge und schuttreiche Wildbachausbrüche vor.

So konnten in der Zeit von 1925 bis 1956 in den Nordalpen insgesamt 73 Felsund Bergstürze und 118 Erdrutschungen festgestellt werden, die sich an 101 Regentagen ereigneten. Diese Gesamtzahl von 191 Fällen von Erd- und Felsbewegungen verteilt sich wie folgt auf die Hauptgebiete der Nordalpen:

Berner und Freiburger Alpen.

Erdrutsche Felsstürze Regentage

Vierwaldstätter-, Glarner- und St. Galler Alpen .

Total

\begin{tabular}{rrr}
52 & 30 & 48 \\
66 & 43 & 53 \\
\hline 118 & 73 & 101
\end{tabular}

Diese große Anzahl von Erdbewegungen ist nicht nur auf die häufigen und starken Regenfälle, die in den Nordalpen namentlich während den Sommermonaten auftreten, sondern auch durch die verwickelte geologische Struktur bedingt; denn bekanntlich sind die verschiedenen Überschiebungen im Gebirgsrelief auch durch lebhaften Wechsel von Kalkbänken, Schiefern und Mergelschichten gekennzeichnet. Dementsprechend treten in den Kalkbergen häufig Felsstürze auf, während an den aus Schiefern bestehenden Hängen Erdrutsche und Wildbachausbrüche vorherrschen.

So haben in den Berner und Freiburger Alpen schuttreiche Wildbäche bei Brienz, Merligen, bei Därligen, Leissigen, Reichenbach und im obern Kandertal große Verheerungen angerichtet. Felsstürze und große Rutschungen haben sich 1922 am Mont Arvel bei Villeneuve, 1951 im Zulgtal (Lit. 1), 1952 bei Le Sépey, 1931 im Tröligraben bei Rüschegg (Lit. 8), 1932 an der Teilegg bei Rougemont und 1956 bei Reutigen ereignet.

In der Innerschweiz kamen mehrmals Felsstürze bei Hergiswil am Lopperberg, am Axenberg, bei Flüelen, an der Windgälle bei Silenen und bei Sisikon vor (Lit. 15). Bekannt sind die Wildbachausbrüche der Gr. Schlieren im Sarnertal, und große Erdrutsche haben sich im Trepsental ob Siebnen, bei Altdorf, bei Dallenwil, im Wäggital u. a. O. ereignet.

In den Glarner- und St. Galler Alpen gingen Felsstürze an den Steilufern des Wallensees, am Kilchlistock im Linthtal, im Durnachtal, im Sernftal, bei Neßlau und bei Flums nieder, und durch bedeutende Erdrutsche wurden Mühlehorn, Betschwanden, der Stoß, die Gegend von Altstätten und Lichtensteig heimgesucht.

\section{VORGÄNGE IN DEN SÜDALPEN}

In der Zeit von 1922 bis 1956 haben sich im Bereiche der Walliser Alpen und in ihrer unmittelbaren Nachbarschaft 112 Fälle von Massenbewegungen ereignet, nämlich 83 Erdschlipfe und Murgänge sowie 29 Fels- und Bergstürze. 


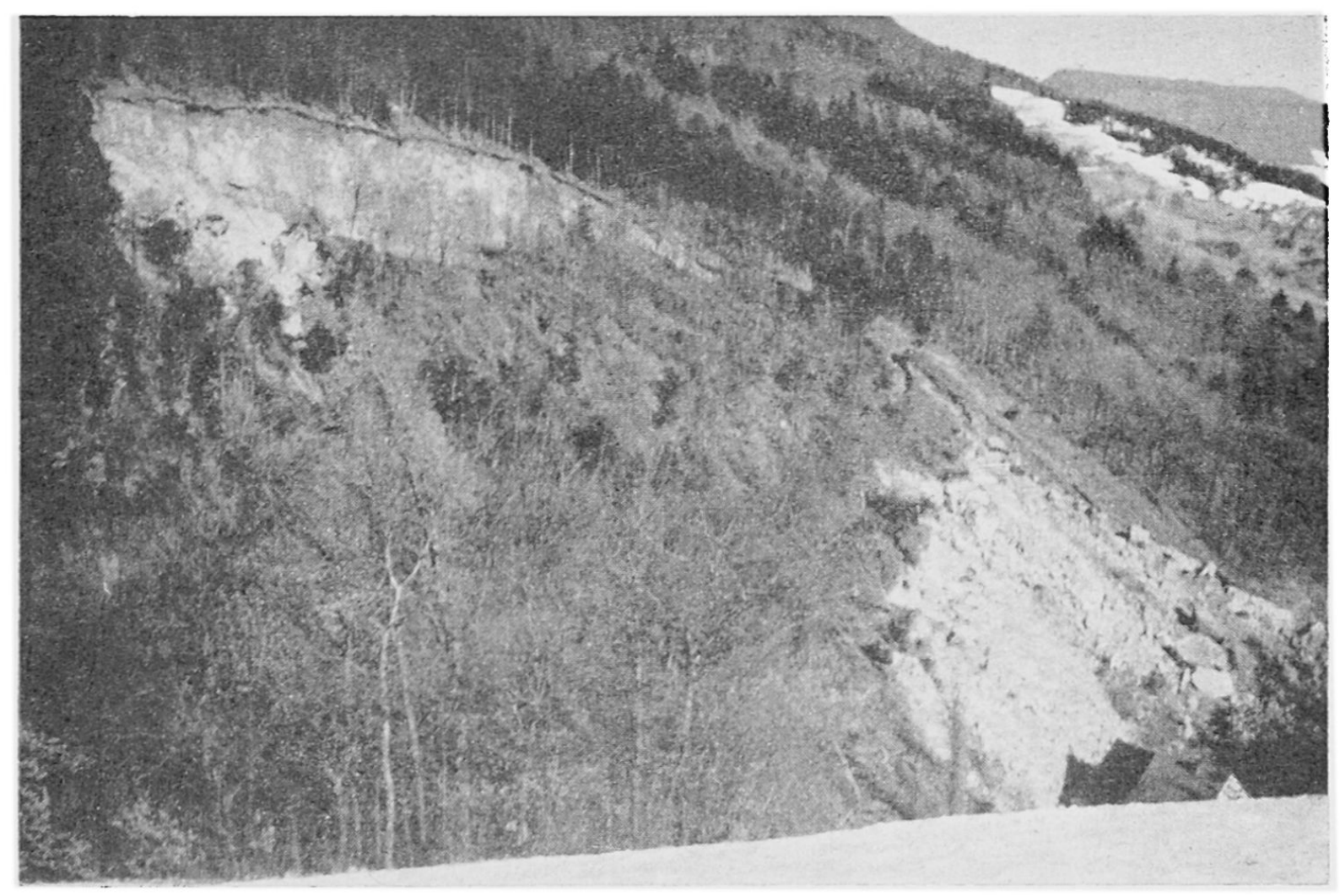

Abb. 1 Felsstur\% an der Egg bei Obererlinsbach (Aargauer Faltenjura) im Februar 1957 Phot. M. Hintermann

In 74 Fällen wurden Erdschlipfe bei regnerischem Wetter festgestellt, wobei sie durch das Vorherrschen von teils schieferigen, teils lockeren Gesteinsarten begünstigt worden sind; diese treten in verhältnismäßig großer Verbreitung namentlich an Berghängen der zahlreichen Täler auf (Lit. 10).

So weisen die südlich des Rhonelängstales in der Hauptschieferzone gelegenen Täler die folgenden Zahlen an stattgefundenen Erdrutschen und Felsstürzen auf: Im Talgebiet der Drance de Bagnes 17, im Ehringertal 9, im Eifischtal 7, im Visptal 8. Im Haupttal kamen zwischen Brig und Martinach 8 größere Rutschungen vor. Auch das in Flysch eingetiefte Val d'Illiez wurde mehrmals durch Erdrutsche heimgesucht, und der seit Jahrhunderten berüchtigte Torrent de Saint Barthélmy hat mehrmals seine geschiebereichen Ausbrüche wiederholt (Lit. 12, 14).

Felsabstürze kommen in ihrer Mehrzahl in höheren Lagen, an Kämmen und Gipfeln vor. In einigen Fällen sind solche durch lokale Erdbeben verursach worden: von diesen sind diejenigen des Jahres 1946 von starken Wirkungen gewesen. Dies war namentlich im Gebiet oberhalb von Ayent und Montana der Fall, wo unter anderem die Alp Sérin zerstört worden ist (Lit. 11).

Ein großer Bergsturz hat im November 1951 auf der Südseite des Simplon bei Vaso stattgefunden, wobei auch die Bahnlinie auf längere Zeit gesperrt wurde und 4 Menschen den Tod gefunden haben.

Ein weiterer Bergsturz, der ebenfalls mehreren Personen den Tod brachte, fand auf der Südseite der Hauptkette im Tal von Valpelline am 9. Juni 1952 statt. Überdies haben durch plötzliche Erdrutsche und Felsstürze im Kanton Wallis noch weitere Personen den Tod gefunden, so 2 am 27. Dezember 1947 bei Morgins sowie je eine Person im Januar 1931 bei Mörel, im September 1927 und im Oktober 1953 im Eifischtal.

Obwohl der Kanton Tessin im ganzen bedeutend mehr Niederschläge erhält als das Wallis, weist er doch eine wesentlich geringere Zahl von Erdbewegungen auf als 


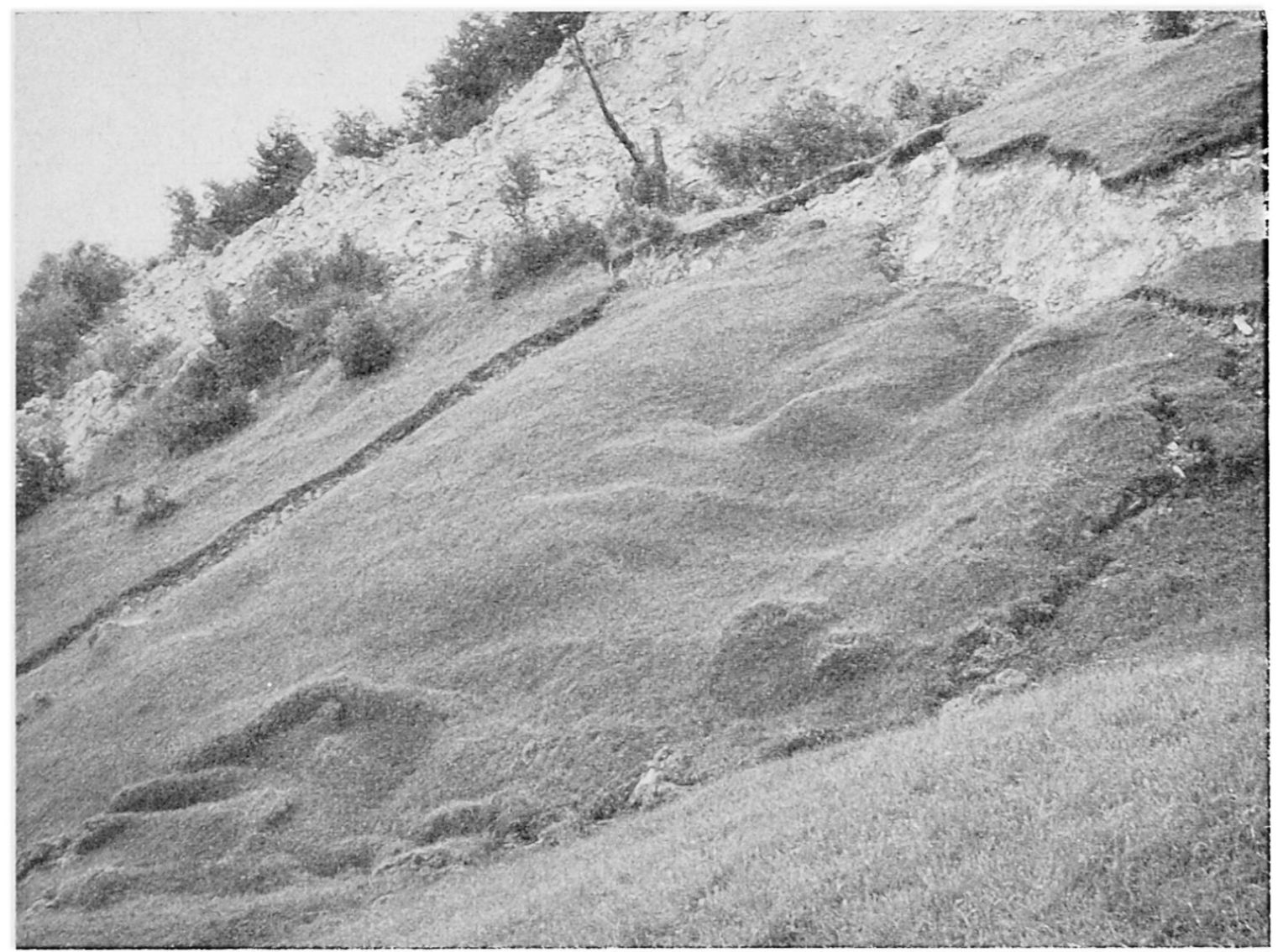

Abb. 2 Bergrutsch von Schuders bei Schiers im Prätigau, eines der aktuellsten Beispiele von Bewegungen großer Erdmassen (rund 22,106 t). Detail aus dem Rutschgebiet, Zustand am 6. 8. 1956. Phot. H. BÖRI.IN, mit freundlicher trlaubnis des Verfassers entnommen aus H. J ̈̈CKLI: (iegenwartsgeologie des bündnerischen Rheingebicts. Bern 1957.

dieses, nämlich 32 Rutschungen und 10 Felsstürze; im ganzen also nur 42 Vorgänge, die sich an 28 Regentagen ereignet haben. Diese geringe Zahl erklärt sich zum einen Teil aus dem fast um die Hälfte kleineren Areal des Kantons Tessin gegenüber dem des Wallis und zum andern Teil aus der verschiedenen geologischen Beschaffenheit, indem im Tessin kristalline widerstandsfähige Gesteine weitaus vorherrschen.

Unter den umfangreichen Erdbewegungen im Kanton 'Tessin sei hier an die Rutschungen bei Campo im Val Maggia sowie an den Bergsturz am Monte Arbino erinnert, welche Vorgänge seiner Zeit von verschiedenen Geologen geschildert worden sind (Lit. 5 [pag. 201], 9, 13, 23).

Auch im Kanton Graubïnden ist trotz dessen Größe die Zahl der im gleichen Zeitraum festgestellten Erdbewegungen wesentlich geringer als im Wallis; sie beträgt insgesamt nur 56; von diesen entfallen 39 auf Erdrutsche und 17 auf Fels- und Bergstürze. Alle diese Vorgänge ereigneten sich an 39 Regentagen, und ihre Verbreitung läßt deutlich eine Bevorzugung der im nördlichen Gebiet vorherrschenden, leicht verwitterbaren Bündnerschiefer erkennen, die nach E. RODER zu den halb durchlässigen Gesteinen gerechnet werden (Lit. 19).

So entfallen auf das Vorderrheintal und seine Seitentäler gegen 20 Erdrutsche; auf das Hinterrheintal, insbesondere auf das Domleschg und das Schams kommen 7 Fälle, ebensoviele auf das Julier- und Albulagebiet; eine gleiche Anzahl weisen das Rheintal unterhalb Chur und das Schanfigg zusammen auf. Im Tal der Landquart wurden 6 Orte genannt, an denen größere Erdrutsche stattgefunden haben. Diesen 47 im Rheingebiet vorgekommenen Erdbewegungen stehen 10 gleiche und ähnliche gegenüber, die sich in dem hauptsächlich aus kristallinen Gesteinen aufgebauten Innund Addagebiet ereignet haben. 


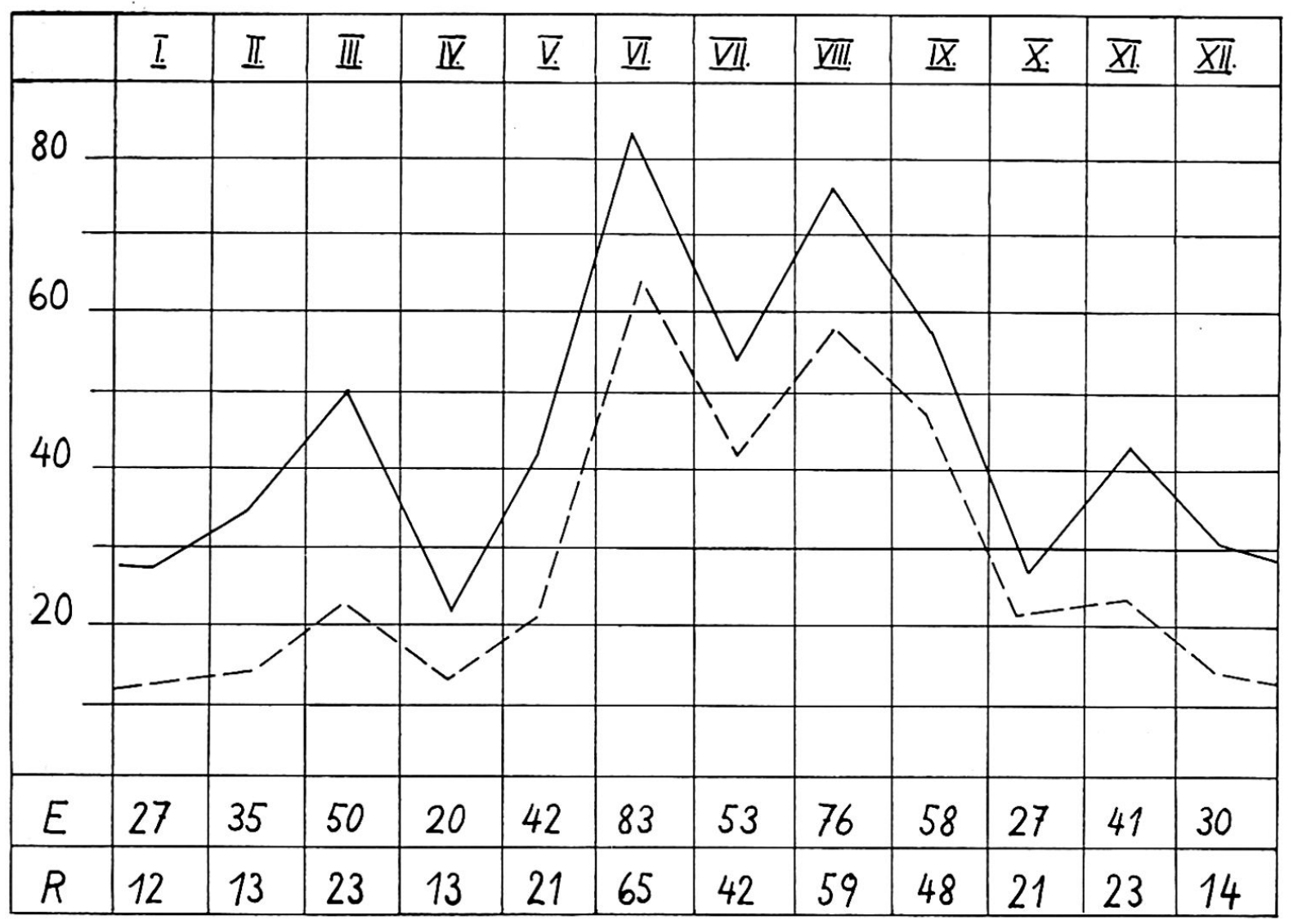

\section{Diagramm der totalen monatlichen Erdbewegungen \\ $\mathrm{E}=$ Anzahl der Erdrutsche, Felsstïrze u. ä. $\mathrm{R}=$ Anzahl der betreffenden Regentage}

Zu den furchtbarsten Ereignissen Graubündens gehört der im April 1939 auf der Südseite des Flimsersteins erfolgte Bergsturz bei Fidaz, wobei über 20 Erwachsene und Kinder eines Ferienheims getötet wurden (siehe N.Z.Z. Nr.636 und «Der Bund»Nr. 167, 1939).

\section{SCHLUSSFOLGERUNGEN}

In meinem Verzeichnis der 544 Erdbewegungen, die sich seit 1920 bis 1956 in unserem Lande ereignet haben, ist überall auch das Datum jener Vorgänge angegeben; es geht daraus hervor, daß die Hälfte derselben in den 4 Monaten Juni-September stattgefunden hat, während sich die andere Hälfte der genannten Vorgänge auf die übrigen 8 Monate verteilt. In den genannten 4 Monaten kamen im Mittel je gegen 70 Erdbewegungen vor, am meisten in den Monaten Juni und August.

Die Monate mit den geringsten Vorfällen, nämlich 20 bis 35, sind drei Wintermonate und der April.

Auf unserer graphischen Darstellung machen auch die Verbindungslinien der während der Beobachtungszeit gezählten Regentage $(R)$ entsprechende Kurven mit: In den eben erwähnten Wintermonaten und im April bleiben sie niedrig, indem sie je nur 12-14 Regentage angeben, während die $Z$ ahl der Regentage entsprechend den Kurven der Erdrutsche ansteigt, und zwar bis auf 65 im Monat Juni und auf 59 im August.

Es darf deshalb wohl auf einen ursächlichen Zusammenhang zwischen Erdbewegungen und Regenfällen geschlossen werden, eine Auffassung, die bereits von andern Geographen geäußert worden ist, und zwar in dem Sinne, daß Erdschlipfe, Murgänge und sogar Bergstürze durch anhaltende Regenfälle verursacht worden sind (Lit. 5 [p. $204 \mathrm{ff}$ ]). Dabei ist zu beachten, daß eine bestimmte Anzahl von Erdbewegungen erst nach vorangegangenen Regenfällen und entsprechender Durchfeuchtung des Bodens eingetreten ist. 
Im Hinblick auf die weitere Tatsache, daß in den Alpen jährlich 200 bis $300 \mathrm{~cm}$ Niederschläge fallen, und zwar davon die größere Menge während der warmen Jahreszeit in Form von Regen, ergibt sich, daß die einzelnen Gebirgsgruppen einer sehr starken Benetzung ausgesetzt sind, die sich je nach dem Grade der Durchlässigkeit der Gesteinsschichten verschieden auszuwirken vermag. So ist allgemein bekannt, daß es in den Kalkbergen zufolge ihrer ausgesprochenen Durchlässigkeit zu einer bedeutenden unterirdischen Entwässerung und derzufolge zu mannigfacher Höhlenbildung kommt. Wo jedoch Gesteine wenig durchlässig sind, wie Schiefer, Mergel und kristalline Felsen, erfahren sie eine allgemein lebhafte Abspülung, die bei starken Regenfällen zu schuttreichen Wildbächen oder zu zahlreichen Rutschungen führt; nicht selten ist es unter solchen Witterungsverhältnissen zu Bergstürzen gekommen.

Felsstürze in der Gipfelzone sind häufig durch den Wechsel winterlicher kalter und darauffolgender wärmerer Witterung verursacht worden. Sie ereignen sich aus diesem Grunde vorzugsweise in den Frühjahrsmonaten. In solchen Fällen stürzen die Felsblöcke auf die Schutthalden herunter, die sich in den eiszeitlichen Karen der Alpen gebildet haben, und zwar sowohl in den Kalkbergen wie auch in den aus kristallinen Gesteinen aufgebauten Gebirgsgruppen (Lit. 15, p. 86, sowie 5, p. 206-210).

Auch in den tieferen Lagen sind Felsstürze niedergegangen und zwar in mehreren Fällen an den steilen Felshängen eiszeitlicher Trogtäler. Solche Vorkomnisse wurden beispielsweise im Berner Oberland bei Meiringen, am Hasliberg, bei Hopflauenen, im Haslital, bei $Z$ weilütschinen, im Lauterbrunnental, im Kandertal, im Justistal u. a. O. beobachtet.

Beispiele weiterer Felsstürze von ähnlicher Art ließen sich auch aus Trogtälern der Kantone Uri, Glarus, Wallis und Tessin nachweisen.

\section{LITERATUR}

1. BeCK, P.: Der Erdschlipf im Eriz (Kt. Bern) 9. Mai 1951. Verh. Schweiz. Nat. Ges. 1952. 2. Billwiler, Maurer u. Hess: Das Klima der Schweiz. Frauenfeld 1909. - 3. Buxtorf, A.: Die Schuttrutschung in der Klus von Court. Nat. Z. 11. VI. 1937 Basel. - 4. Choffat, Ph.: L'écoulement du M. d'Arvel. Bull. Soc. Vaud 1928. - 5. FrûH, J.: Geographie der Schweiz. Bd. 1. St. Gallen 1930. - 6. Heim, A.: Bergsturz und Menschenleben. Nat. Ges. Zürich 1932. - 7. Heim, A.: Geologie der Schweiz. Bd. 1. Leipzig 1919. - 8. v. KäNEL, F.: Errdrutschgebiet «Tröli». Heimatgl. Schwarzenburg 1931. - 9. LehmanN, O.: Die Gestalt der Landschaft d. gr. Massenanhäufungen in der Gemeinde Campo (V. Maggia). Zürich 1934. - 10. Lugeon, M.: Le glissement des hameaux de Montagnon et de Produit, comm. de Leytron (Valais). Bull. Soc. Murith., 1932/33. - 11. MARIETAN, I.: Le tremblement de terre du 25 janvier 1946. Bull. Soc. Murith., 1946. - 12. MoNTANDoN, F.: Le torrent du Mauvoisin et la vallée de St-Maurice (Valais). Mat. Etud. d. Calam. $\mathrm{N}^{\circ} 27$, 1932. - 13. Montandon, F.: Les éboulements du M. Arbino. Ebendort 1928. - 14. Montandon, R.: Chronique des calamités récentes. Ebendort 1925. - 15. Nussbaum, F.: Die Täler der Schweizeralpen. Bern 1910. - 16. Penck, A. und Brǘckner, E.: Die Alpen im Eiszeitalter. Leipzig 1909. 17. Oechslin, M.: Berichte der Naturforsch. Gesellschaft Uri, 1933. - 18. Peter, A.: Le glissement de terrain dans les gorges de Moutier en 1937. Les Int. économ. du Jura, Delémont 1938. 19. Roder, E.: Niederschlag und Abflu $B$ im bündner. Rheingebiet 1894-1904. Mitt. Landeshydrogr. 1914. - 20. Schwabe, E.: La structure géologique de la Gorge de Moutier. Tribune de Genève, 1937. - 21. SuTER, P.: Beiträge zur Landschaftskunde des Ergolzgebietes. Basel 1926. - 22. Vosseler, P.: Der Aargauer Jura. Aarau. - 23. Zurbuchen, M. u. KNoblauch, P.: Die Bewegungen am Monte d'Arbino bei Bellinzona. Les Alpes, 1927.

\section{NOTE SUR DES GLISSEMENTS ET DES ÉBOULEMENTS RÉCENTS EN SUISSE}

Se basant sur des descriptions de géologues et de géographes suisses, puis sur les notes publiées dans les "Matériaux pour l'étude des Calamités" et sur un grand nombre d'indications dans les journaux, l'auteur a constaté que depuis 1920544 cas de glissements de terrain, de torrents de lave, de chûtes de pierres et des éboulements ont eu lieu; en 361 cas, ces événements ont été causés par de journées de pluies ou de mauvais temps.

En voici la distribution de ces accidents dans les différentes parties de notre pays: 


\begin{tabular}{|c|c|c|}
\hline Région & glissements & $\begin{array}{l}\text { chûtes de pierres } \\
\text { ou éboulements }\end{array}$ \\
\hline . . . . & 40 & 4 \\
\hline Plateau . . . & 89 & 10 \\
\hline Alpes du côté $\mathbf{N}$ & 118 & 73 \\
\hline Alpes du côté $S$ & 154 & 56 \\
\hline
\end{tabular}

Dans la plupart des régions attaquées, la constitution géologique joue un rôle considérable. D'autre part, on a constaté que les accidents en question sont deux fois plus fréquents et nombreux dans les mois d'été qui sont très pluvieux, que pendant les mois des saisons fraiches et froides de l'hiver et du printemps. (Voir la figure: Diagramme).

\title{
ZUR GLIEDERUNG NEPALS IN NATUR- UND BEVÖLKERUNGSGEBIETE
}

\author{
Toni Hagen
}

Nepal war bis 1950 für Fremde ein praktisch verbotenes Land. Die wenigen Besucher, die von den damaligen Maharajas der Rana-Dynastie ein Einreisevisum erhielten, mußten sich auf die Hauptstadt beschränken. Einzig die Angehörigen der britischen Gesandtschaft hatten etwas Bewegungsfreiheit, die sie denn auch intensiv für botanische und zoologische Forschungen benutzten. Abgesehen von den in der Hauptstadt lebenden Newari war Nepal vom ethnologischen Standpunkt aus unbekannt.

Es blieb der Abteilung für Orientalistik der Londoner Universität vorbehalten, mit der systematischen Erforschung der ethnologischen Verhältnisse zu beginnen. Seit mehreren Jahren sind zahlreiche ihrer Kapazitäten in Nepal tätig, und mit großer Erwartung darf man ihren Veröffentlichungen entgegensehen.

Die folgende Abhandlung versucht eine erste Übersicht über die verschiedenen nepalischen Bevölkerungsgruppen und ihre Verteilung zu geben. Von Haus aus Geologe, hat es der Verfasser als seine Pflicht erachtet, auch den ethnologischen Belangen etwas nachzugehen. Hat doch bisher niemand außer ihm das gesamte Land bereist.

\section{NATURGEBIETE}

Nepal besitzt große landschaftliche Mannigfaltigkeit. Diese ist primär durch die starke vertikale Gliederung bedingt. Das nepalische Territorium reicht von der Gangesebene (200 m ü. M.) über die Hauptkette des Himalaya (über $8000 \mathrm{~m}$ ) bis in das Tibetische Hochplateau hinein $(4-5000 \mathrm{~m})$. Die Gegensätzlichkeit des Klimas bestimmen besonders zwei Faktoren: die Höhenunterschiede und die Lage zu Kontinent und Ozean, die sich in der ausgesprochenen Zweiteilung des Jahres in die feuchte Sommermonsun- und Trockenzeit äußert. Der Monsun dauert von Anfang Juni bis Ende September. Die achtmonatige Trockenzeit wird durch geringfügige gewittrige Niederschläge anfangs Januar etwas aufgelockert (sog. Wintermonsun).

Diesen Naturgrundlagen entspricht eine deutliche Gliederung von Nepal in sechs natürliche Großregionen :

1. der Terrai-Belt (= Sumpfstreifen), 2. die Siwaliks (entsprechend der Schweiz. Molasse am Alpenrand), 3. die Mahabharatkette (entsprechend der Alpenrandketten, Säntis z. B.), 4. die Midlands (Nepalisches Mittelland), 5. die Himalaya Hauptkette, 6. das Tibetische Plateau,

Mit Terrai-Belt wird der Anteil Nepals an der Gangesebene bezeichnet. Er liegt durchschnittlich $200 \mathrm{~m}$ ü. M. und bildet einen bis zu $45 \mathrm{~km}$ breiten Streifen von der indischen Grenze bis zum Fuß der Siwalikhügel. Östlich des Narayani Flusses allerdings folgt die indische Grenze dem südlichsten Kamm der Siwalikkette auf eine Länge von ca. $70 \mathrm{~km}$. Der Terrai-Belt ist zufolge der Sümpfe und des feucht-heißen Klimas eine wahre Fieberhölle. Trotzdem ist der waldlose 'Teil dicht bevölkert, vorwiegend mit Indern, die ihr Siedlungsgebiet schrittweise nach Norden vorschieben. Der Nordteil dagegen ist mit dichten Urwäldern bedeckt, in denen noch große Mengen von wilden Tieren hausen, wie Tiger, Leoparden, Braunbären, Büffel, Rhinozeros 